THE EFFECTIVE TEMPERATURE OF Am STARS

\title{
C. MEGESSIER
}

Observatoire de Paris-Meudon, 92195 Meudon, France

C. van 't Veer

Observatoire de Paris, 61 av de l'Observatoire, 75014 Paris, France

\begin{abstract}
The values of the effective temperature derived for Am stars by the infra-red flux method and from the Balmer line profiles are in very good agreement. They are hotter by more than $300 \mathrm{~K}$ than the $\mathrm{T}_{\text {eff }}$ given by Lane and Lester (1984) and cooler by about $200 \mathrm{~K}$ than those obtained by Dworetsky et al (1986).
\end{abstract}

\section{INTRODUCTION}

Confident values of stellar effective temperatures are needed to get accurate values of the physical parameters for the chemically peculiar stars : gravity, microturbulence, abundances. They are specially required to interpret the Am phenomenon by diffusion mechanism. Up to now the problem pointed out by Dworetsky and Moon (1986) is not worked out. Discrepancies up to $800 \mathrm{~K}$ exist between $\mathrm{T}_{\mathrm{eff}}$ of Am stars derived by Lane and Lester (1984) and those obtained by Dworetsky and Moon (1986) and van 't Veer et al. (1985). The former group compared the observed energy distribution and the Kurucz one's. The second group used photometric index calibrations and van 't Veer et al. performed a detailed spectral analysis.

In 1990 the Am star 63 Tau (see table 1) was chosen to compare the $T_{\text {eff }}$ derived by two independant methods. The comparison of observed and computed Balmer line profiles led to $T_{\text {eff }}=7300 \mathrm{~K}$ (van 't Veer et al 1990). Through the infra-red flux method Mégessier et al. (1990) found $\mathrm{T}_{\text {eff }}=7160 \mathrm{~K}$ $+/-70 \mathrm{~K}$. Since one has to take into account the small effect of a red companion on the red flux, this value has to be raised by about $100 \mathrm{~K}$ or less. The two methods lead to $T_{\text {eff }}$ values which are in agreement within $140 \mathrm{~K}$ (or less) which is almost in the error bars. Moreover these $T_{\text {eff }}$ are intermediate between $7000 \mathrm{~K}$ given by Lane et al. (1984) and $7570 \mathrm{~K}$ given by Dworetsky et al. (1986). Table 1 summarises the effective temperatures obtained previously for $63 \mathrm{Tau}$.

Then we decided to go further and to derive new effective temperatures through both methods for some others Am stars. First results are given here for 81 Tau and $\tau$ UMa. 


$$
\mathrm{T}_{\text {eff }} \text { of Am Stars }
$$

TABLE 1 History of the effective temperature determination of 63 Tau

\begin{tabular}{lc}
\multicolumn{1}{c}{ Authors } & $\mathrm{T}_{\text {eff }}(\mathrm{K})$ \\
\hline & \\
van 't Veer et al (1985) & 7750 \\
Lane and Lester (1984) & 7000 \\
Dworetsky Moon.(1985) & 7570 \\
van 't Veer et al (1990) & 7300 \\
Mégessier et al (1990) & $7160 \leq 7290$ \\
& (companion) \\
\hline
\end{tabular}

\section{NEW $T_{\text {eff }}$ DETERMINATION FOR THE Am STARS 81 Tau and $\tau$ UMa}

The $\mathrm{T}_{\text {eff }}$ of the two stars were determined through the IRFM. The $\mathrm{T}_{\text {eff }}$ of $\tau \mathrm{UMa}$ was also obtained from the Balmer line profiles. The only but important change since the 1990 determinations is the use of Atlas 9 in which a large amount of element transitions have been introduced.

\section{Comparison of the observed and theoretical Balmer line profiles}

The Am star $\tau$ UMa was observed with a CCD detector at the spectrograph Aurélie (OHP) to obtain Balmer line profiles, namely $\mathrm{H}_{\alpha}$ and $\mathrm{H}_{\beta}$. A first study by comparison with Atlas $\mathbf{8}$ theoretical profiles lead to an effective temperature between 7000 and $7100 \mathrm{~K}$. The comparison with Atlas 9 models is in progress and we can already expect a $\mathrm{T}_{\text {eff }}$ increase of about $100 \mathrm{~K}$. This is due to the larger blanketing effect in Atlas 9.

\section{The infra-red flux method}

The infra-red flux method was used already by Mégessier (1988) for normal and peculiar A type stars. The required data are the observed total integrated flux and one or more IR monochromatic fluxes. We used IUE low resolution spectra in the UV and the visible fluxes measured by Lane and Lester (1984). Carney (1982) gives the IR photometric colors of 81 Tau. The colors J and K, as well as the visible flux, were transformed in absolute flux with Hayes calibration (1969). Since no IR photometric data are available for $\tau \mathrm{UMa}$, the monochromatic fluxes at $\lambda=1.04 \mu \mathrm{m}$ and $\lambda=1.08 \mu \mathrm{m}$ as given by Lane and Lester were used.

The theoretical ratios between the total integrated flux and the IR monochromatic flux, as a function of the effective temperature, were computed with the new Atlas 9 models. The resulting effective temperatures as deduced from $\mathrm{J}$ and $\mathrm{K}$ fluxes are shown in table 2 . The $\mathrm{T}_{\text {eff }}$ of $63 \mathrm{Tau}$ was also derived with Atlas 9, from the observed ratio used earlier with Atlas 8 (table 2). Note that in 1990 the $\mathrm{T}_{\text {eff }}$ deduced with Atlas 8 models from the $\mathrm{K}$ flux only was 
given. In fact the mean between $T_{\text {eff }}(J)=7050 \mathrm{~K}$ and $T_{\text {eff }}(K)=7170 \mathrm{~K}$ is 7110 $\mathrm{K}$.

The $T_{\text {eff }}$ increase found when using Atlas 9 instead of Atlas 8 is logically explained by the backwarming effect in the infra-red flux induced by the heavy blanketing in the short wavelength range (Mégessier 1992)

TABLE $2 \mathrm{~T}_{\text {eff }}$ of 63 Tau by the IRFM

$\mathrm{T}_{\mathrm{eff}}(\mathrm{J})(\mathrm{K}) \quad \mathrm{T}_{\mathrm{eff}}(\mathrm{K})(\mathrm{K})$

$\begin{array}{ccc}\text { Atlas 8 } & 7050 & 7160 \\ \text { Atlas 9 } & 7110 & 7270 \quad \leq 7350 \\ & & \end{array}$

\section{DISCUSSION}

The $\mathrm{T}_{\text {eff }}$ of the three stars studied here, as derived through the four methods recalled in this paper, are gathered in table 3 . The presence of a red companion for two of the stars implies a correction which was estimated to be less than $150 \mathrm{~K}$. The corrected $\mathrm{T}_{\text {eff }}$ are given in the last column.

First we can see that the $T_{\text {eff }}$ derived either through the infra-red flux method or by comparing the observed Balmer line profiles with the theoretical one's are in agreement within $100 \mathrm{~K}$. Moreover for the three stars the behaviour is the same. Lane et al (1984) found $\mathrm{T}_{\text {eff }}$ systematically cooler by about $300 \mathrm{~K}$ than those we have obtained, while Dworetsky et al (1986) give $T_{\text {eff }}$ hotter by about the same amount.

Hauck and North (1992) provided us for the $T_{\text {eff }}$ deduced from recent calibrations of the Geneva photometric indices (B2-G) and (B2-V1), based on individual effective temperature determinations. These values are shown in table 4 , col 1 and 2 and compared to our values (col 3 and 4). The (B2-G) index is influenced by the presence of a red companion, whereas (B2-V1) is not. While for $81 \mathrm{Tau}$, which is a simple star, the three $\mathrm{T}_{\text {eff }}$ are are in a fair agreement, for $\tau \mathrm{UMa}$, which has a red companion, $\mathrm{T}(\mathrm{B} 2-\mathrm{V} 1)$ is in better agreement with our determination than $\mathrm{T}(\mathrm{B} 2-\mathrm{G})$.

In its principle the infra-red flux method is very close to the effective temperature definition so that we think it is a good reference. In the temperature range considered, the Balmer lines are very sensitive to the effective temperature and they are not affected by the superficial gravity. Then we think that our $\mathrm{T}_{\mathrm{eff}}$ can be considered as the closest to the actual effective temperatures of Am stars. 


$$
\mathrm{T}_{\text {eff }} \text { of Am Stars }
$$

TABLE 3 Effective temperature (in K) of Am stars through four methods

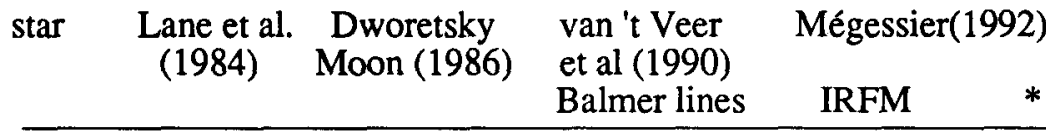

$\begin{array}{lccccc}63 \text { Tau } & 7000 & 7570 & 7300 & 7190+/=80 & \leq 7350 \\ 81 \text { Tau } & 7300 & 7800 & & 7615+/=70 & \\ \tau \text { UMa } & 6600 & 7390 & 7100 & 7050+/ \_80 & \leq 7200\end{array}$

* $\mathrm{T}_{\text {eff }}$ corrected from the red companion effect

TABLE 4 Comparison with Geneva photometric indices calibration $\mathrm{T}_{\mathrm{eff}}$ in $\mathrm{K}$

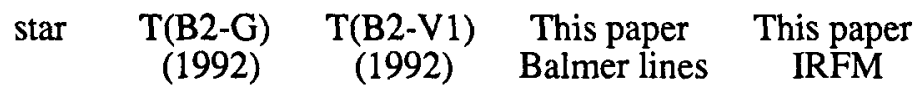

$\begin{array}{llllr}63 \text { Tau } & 7287 & 7400 & 7300 & \leq 7350 \\ 81 \text { Tau } & 7594 & 7620 & & 7615 \\ \tau \text { UMa } & 7023 & 7110 & 7100 & \leq 7200\end{array}$

\section{REFERENCES}

Dworetsky, M.M., Moon, T.T , 1986, Mon. Not. R. Astr. Soc., 220, 787 Hauck, B., North,P. 1992 (private communication)

Hayes, D.S., 1979, Dudley Obs. Reports,14, 297

Lane, M.C. and Lester, 1984, Astrophys. J., 281, 723

Mégessier, C., 1992, Astron. Astrophys. (submitted)

Mégessier, C., 1988, Astron. Astrophys. Suppl., 72, 551

Mégessier, C., van 't Veer C., 1990, poster presented at the IAU Symposium $\mathrm{N}^{\circ} 145$, "Evolution of stars : The photospheric abundance connection", Bulgaria, Eds : Michaud, G., Tutukov, A., Bergevin, M., p. 35

van 't Veer, C., Coupry, M.F., Burkhart, C., 1985, Astrn. Astrophys.,146, 139

van 't Veer, C., Cayrel, R., Coupry, M.F., 1990, poster presented at the IAU Symposium $\mathrm{N}^{\circ} 145$, "Evolution of stars : The photospheric abundance connection", Bulgaria, Eds : Michaud, G., Tutukov, A., Bergevin, M., p. 35 


\section{DISCUSSION (Mégessier \& van't Veer)}

MÉGESSIER: Smalley and Dworetsky presented a poster at this colloquium on the $T_{\text {eff }}$ of $A, F$, and Am stars. They used four different methods. The final values they give for two Am stars in our study are larger than ours by $150 \mathrm{~K}$ and $200 \mathrm{~K}$ :

$\begin{array}{lcc} & \text { Mégessier \& van't Veer } & \text { Smalley \& Dworetsky } \\ 63 \text { Tau } & \lesssim 7350 & 7500 \\ 81 \text { Tau } & 7615 & 7650 \\ \tau \text { UMa } & \lesssim 7200 & 7400\end{array}$

MISSANA: It is possible that A-type stars have a quite thick envelope (as I have obtained from some measurements of the Compton effect). Then, a spread of the values of temperature of the stars can be avoided by taking into account the presence of a scattering layer around the star.

MÉGESSIER: The effect of a layer around the star affects the IR flux at longer wavelengths than those used here ( $\mathrm{J}$ and $\mathrm{K}$ bands), so the $\mathrm{T}_{\text {eff's }} \mathrm{s}$ we have derived are clear of such an effect.

COWLEY: Of course the effective temperature is important from the point of view of the iron-peak abundances. Is it important whether the iron is overabundant or normal?

MÉGESSIER: Yes it is. A good $T_{\text {eff }}$ is required to get good metal abundances. But here we are interested in the $T_{\text {eff }}$ determination. The iron, as well as the metal abundances, play an important role. Their influence on the Balmer line profiles has been shown already for 63 Tau (van't Veer et al., 1990). The profiles computed with solar metal abundance models lead to $T_{\text {eff }}=6400 \mathrm{~K}$ whereas $3 \times$ solar gives $T_{\text {eff }}=6300 \mathrm{~K}$. The $T_{\text {eff }}$ given by the IRFM is metal-abundance dependent in a similar way (Mégessier 1992).

LODÉN: Could you please indicate the uncertainty in the five temperature estimates at the top so we can see to what extent the differences are significant?

MÉGESSIER: Even when the uncertainties are considered the differences in $T_{\text {eff }}$ remain real. We have gathered the $T_{\text {eff }}$ obtained so far for 63 Tau to point out the convergence of the $T_{\text {eff }}$ value when accurate observations and the physically best-grounded methods are used. (The detailed analysis performed in 1985 was based on photographic plates whereas now the Balmer lines are obtained with a CCD.) 